Eric D. Ragan*

Ajith Sowndararajan

Regis Kopper

Doug A. Bowman

Center for Human-Computer

Interaction

Department of Computer Science

Virginia Tech

Blacksburg, VA 24060

\section{The Effects of Higher Levels of \\ Immersion on Procedure \\ Memorization Performance and \\ Implications for Educational \\ Virtual Environments}

\begin{abstract}
Researchers have proposed that immersion could have advantages for tasks involving abstract mental activities, such as conceptual learning; however, there are few empirical results that support this idea. We hypothesized that higher levels of immersion would benefit such tasks if the mental activity could be mapped to objects or locations in a 3D environment. To investigate this hypothesis, we performed an experiment in which participants memorized procedures in a virtual environment and then attempted to recall those procedures. We aimed to understand the effects of three components of immersion on performance. The results demonstrate that a matched software field of view (SFOV), a higher physical field of view (FOV), and a higher field of regard (FOR) all contributed to more effective memorization. The best performance was achieved with a matched SFOV and either a high FOV or a high FOR, or both. In addition, our experiment demonstrated that memorization in a virtual environment could be transferred to the real world. The results suggest that, for procedure memorization tasks, increasing the level of immersion even to moderate levels, such as those found in head mounted displays (HMDs) and display walls, can improve performance significantly compared to lower levels of immersion. Hypothesizing that the performance improvements provided by higher levels of immersion can be attributed to enhanced spatial cues, we discuss the values and limitations of supplementing conceptual information with spatial information in educational VR.
\end{abstract}

\section{Introduction}

Virtual reality (VR) technologies have been used successfully for a variety of applications to facilitate learning of real-world activities and procedures. Such applications, including many of those used for vehicular operation training, military simulations, and medical operations training, often employ immersive VR systems in which the virtual environment (VE) appears to surround the user in space. Applications in these domains take advantage of the physical, wholebody interactions provided by such systems. For example, flight simulators make use of a real, physical cockpit so that pilots-in-training can use the actual controls to fly the simulated airplane (F. Brooks, 1999). Similarly, laparoscopic

\footnotetext{
*Correspondence to eragan@vt.edu.
} 
surgery simulators use high-fidelity haptic devices to help physicians learn the necessary motor skills before operating on real patients (Botden, Buzink, Schijven, \& Jakimowicz, 2007).

Other types of applications take advantage of immersive VR's higher-quality and more realistic spatial cues (e.g., stereoscopy, motion parallax), which makes it possible to provide users with higher levels of spatial understanding than could be achieved using traditional displays. For instance, vehicle designers have long used immersive VR systems to better understand their designs before they are built (F. Brooks, 1999). Scientists use immersive technologies to visualize complex 3D structures and data sets (van Dam, Forsberg, Laidlaw, LaViola, \& Simpson, 2000). Engineers plan underground features, such as oil wells, using immersive VR (Lidal, Langeland, Giertsen, Grimsgaard, \& Helland, 2007).

While the reasons for the success of these two sets of VR applications are understood, other proposed applications, such as educational applications, do not fit within these categories. Educational VR systems have been developed for the purpose of helping students to learn conceptual information and principles. For example, researchers have prototyped immersive VR systems for mathematics education (Kaufmann, Schmalstieg, \& Wagner, 2000; Roussou, Oliver, \& Slater, 2006) and for learning complex principles of physics (Dede, Salzman, \& Loftin, 1996). We can characterize these applications as interactive visualizations for the purpose of conceptual learning, in which abstract concepts or very large- or small-scale phenomena are mapped to human-scale visual representations. But it is not known if immersive VR technology is necessary or beneficial for such learningbased applications or if standard, nonimmersive displays would work just as well. Furthermore, it is not clearly understood what features of VR are beneficial for what educational purposes (Dede et al., 1996; Salzman, Dede, Loftin, \& Chen, 1999). Greater knowledge of how various features of immersive VR support different levels of cognitive processing is needed to understand how to effectively design VR applications that are conducive to learning activities.

Evaluating how different components of immersion affect learning is a difficult challenge, particularly because measurement of conceptual learning is not well understood and is subject to many potential biases. Bloom, et al. (1956) and Krathwohl (2002) explain how knowledge can be considered in terms of different levels of understanding and mastery. For example, factual knowledge, knowledge of how to perform tasks based on learned methodology, and an understanding of how new information is related to previously learned information, can be thought of as different levels of learning. Different pedagogical approaches can be used depending on the types of educational objectives instructors hope to achieve (Krathwohl). Similarly, different types of assessments can be used for evaluations, though it is uncertain what evaluation methods are the best for different situations (Kennedy, 1999).

Rather than attempt the unwieldy evaluation of conceptual learning directly, we use a memorization task as a more manageable example of a mental activity that still requires the transfer of information from a VE to a user. As knowledge and recollection of facts is considered to be a simple, foundational stage of the learning process (Bloom et al., 1956; Krathwohl, 2002), supporting such activities can reinforce the deeper levels of learning that are desired in educational applications. We have conducted a study of the use of VR technology for a procedure memorization activity. In this task, a user in a VE is shown a procedure involving several steps/actions and is asked to rehearse and memorize the procedure. This task requires the perception and memorization of abstract information; thus it is a simple approximation of conceptual learning.

We performed this study within a theoretical framework centered on the concept of immersion. Following Slater (2003), we define immersion as "the objective level of fidelity of the sensory stimuli produced by a VR system." In other words, immersion depends only on the technology used to produce the $\mathrm{VE}$, and is not necessarily related to the user's experience of the VE (i.e., the sense of presence). Immersion can be modified, controlled, and used as an independent variable for empirical studies. With this definition, we can speak of levels of immersion, rather than using terms such as nonimmersive and immersive VR. Furthermore, we note that the overall level of immersion is made up of many compo- 
nents, such as field of view, resolution, and stereoscopy (Bowman \& McMahan, 2007).

We hypothesized that higher levels of immersion would lead to better performance on the procedure memorization task if the procedure could be mapped to spatial locations in the VE-that is, if the VE could be used as a cognitive aid during learning and recall. Our research supports this hypothesis, showing that particular components of immersion (or combinations of components) are particularly beneficial. This is a first step in demonstrating the benefits of immersion for abstract mental activities, such as conceptual learning, and in determining which VR technologies should be used for such applications. In this paper, as an extension of our original presentation of this study (Bowman, Sowndararajan, Ragan, \& Kopper, 2009), we provide a more detailed description of this research and elaborate on the discussion of its implications for educational VR.

\section{Related Work}

Many researchers have explored procedural training and conceptual learning applications of VR. For example, several projects have explored whether users can learn a procedure through interaction with a virtual agent in a VE (Johnsen et al., 2005; Johnson \& Rickel, 1997; Ponder et al., 2003). Others have hypothesized that content will be more memorable if students experience it firsthand in an immersive VE (Allison \& Hodges, 2000; Salzman et al., 1999). Despite the many educational applications that take advantage of VR technology, few projects have attempted to formally quantify the benefits. Johnson, Moher, Ohlsson, and Leigh (2001) worked to integrate VR systems into an elementary school to help students attain greater understandings of scientific concepts, but were unable to find a meaningful method for comparing comprehension levels to those achieved with traditional instructional methods. Roussou et al. (2006) compared test results for groups of young students using either their Virtual Playground or a physically similar exercise to learn about mathematical fractions, finding no meaningful quantitative differences between their physical and VR exercises. Bowman, Hodges, Allison, and Wineman (1999) found evidence for learning improvements for students who used a VR application to aid their classroom study of zoo habitat design, but the researchers were unable obtain statistical significance due to small class size and poor attendance.

Similar to our study of the effects of components of immersion, the ScienceSpace project (Dede et al., 1996) studied the benefits of groups of features of VR for three different applications. For one of these applications, MaxwellWorld, an application for learning about electric fields, the researchers found significant improvements over more traditional methods (Dede, Salzman, Loftin, \& Sprague, 1999). While this was an important step in evaluation, it was not possible to determine the values of the individual components of immersive technologies. The results of this study did suggest that the ability to view the virtual world through multiple viewpoints, a useful method for achieving a better understanding of the $3 \mathrm{D}$ space, was an important contributor to improved learning within the VE. This serves as evidence of the importance of strong spatial cues in certain learning situations.

In a study related to the memorization of object information, Mania, Robinson, and Brandt (2005) found evidence that object recognition was significantly better with higher rendering quality. While other studies have investigated the effects of various components of immersive VR (Pausch, Proffitt, \& Williams, 1997; Mania, Troscianko, Hawkes, \& Chalmers, 2003), as well as interaction techniques (e.g., B. Brooks, Attree, Rose, Clifford, \& Leadbetter, 1999), on memorization of spatial layouts of objects, these studies focused on the effects on memorization of spatial location rather than on the memorization of additional information.

Placing greater emphasis on learning new information that is not bound to the specifics of the VE, our previous research (Sowndararajan, Wang, \& Bowman, 2008) found that users performed significantly better in a procedural memorization task when they used a more immersive VE. The experiment compared a laptop display (low immersion) to a large two-wall projection display (high immersion). Users were shown a medical treatment procedure consisting of multiple steps and asked to view, rehearse, and memorize the procedure before recalling it in the VE. Such a mental activity is a 
simplified version of conceptual processing involving perception and memorization, but not necessarily understanding.

Essential to the formation of our hypothesis is the idea that higher levels of immersion provide stronger spatial cues that can help improve spatial understanding. Numerous past studies have provided evidence supporting this claim. For example, Ware, Arthur, and Booth (1993) and Ware and Mitchell (2005) found that head tracking and stereoscopy helped participants to better understand 3D graph structures. Additionally, Schuchardt and Bowman (2007) showed that the addition of stereoscopic vision, head tracking, and increased FOR improved the understanding of complex, underground cave systems. Further, a study by Arns, Cook, and CruzNeira (1999) demonstrated performance benefits of a high-immersion CAVE (CAVE Automatic Virtual Environment) over a low-immersion desktop display for structural detection tasks in statistical visualizations.

While our previous study in procedure memorization (Sowndararajan et al., 2008) did not evaluate participant strategy, we hypothesized that better performance resulted from an increased ability to use a spatial memorization strategy in the high-immersion condition. Since it is still unknown what components of immersion effectively improve procedure memorization, whether higher levels of immersion also improve memorization of more abstract, nonspatial procedures, and whether such learning transfers to the real world, we addressed these questions with the experiment presented in the following section.

\section{Experiment}

We conducted a controlled study to further investigate the effects of immersion on procedure memorization and to determine which components of immersion were responsible for any effects observed in our prior experiment (Sowndararajan et al., 2008).

\section{I Hypotheses}

Our overall hypothesis was that a learning environment with a higher level of immersion would produce better performance in the procedure memorization task. The rationale for this hypothesis is based on the enhanced spatial cues provided by higher levels of immersion-cues resulting from display characteristics such as high FOV, allowing the user to see more of the environment at any one time, and high FOR, allowing the user to make use of natural head and body movements to view other parts of the environment.

Knowing that enhanced spatial cues in higher levels of immersion can lead to improved spatial understanding (e.g., Schuchardt \& Bowman, 2007; Ware \& Mitchell, 2005), our hypothesis was motivated by the idea that spatial memory could be used as a substitute for procedural memory during memorization of a procedure in a VE. In other words, if the steps of the procedure can be mapped to objects or spatial locations, the learner can associate the procedure with these locations in spatial memory. The steps can then be recalled by referencing the spatial locations. Thus, the VE acts as a cognitive aid for the learner. It follows, then, that a learning environment with better spatial cues (a higher level of immersion) should result in better recall performance than a learning environment with impoverished spatial cues (lower level of immersion).

This idea of using spatial locations to aid memory is not new; in fact, it has been used as a memorization technique since classical times. In the method of loci, one memorizes a speech, story, or list by associating each element with a physical or imagined location in a large space, and rehearsing the items while physically or mentally walking through this space (Ericsson, 2003; Yates, 1974). Our contribution to this idea is to use a VE as a replacement for the physical or imagined space used in the classical method.

Regardless of the nature of the space, if the spatial information can be remembered without interfering with the storage of other information, it is theorized that it may be able to aid in the memory of other, nonspatial information (Baddeley, 1998). Past work has provided evidence for this concept, showing performance benefits when supplementing information with spatial location information. Hess, Detweiler, and Ellis (1999) demonstrated that correlating object information with distinct locations inside a grid layout improved participants' abil- 

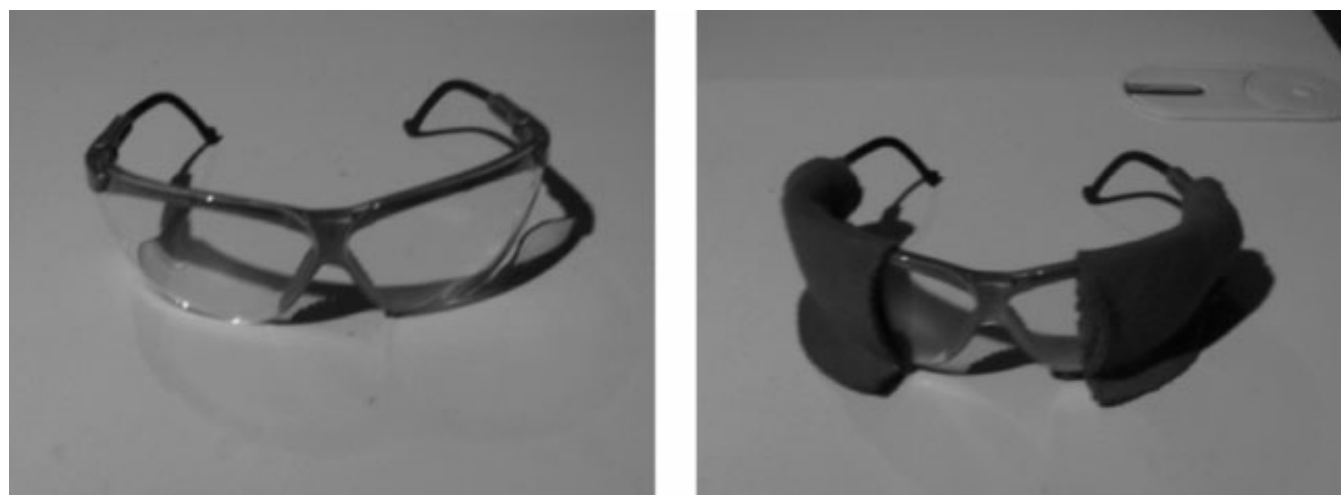

Figure I. (Left) Unblocked goggles used in high FOV conditions; (right) goggles with blinders used in low FOV conditions.

ity to keep track of recent object changes. In a similar sense, in our previous study (Sowndararajan et al., 2008), we suspect that the observed performance gains can be attributed to the enhanced spatial cues available in the high-immersion condition; however, from this study alone, it was not possible to deduce which immersive component (or combination of components) resulted in the observed difference between the two conditions. The two conditions (low and high-immersion) differed in at least the following ways.

- Field of view (FOV; the angular area in the physical world within which the user can see the virtual world at any instant in time).

- Software FOV (SFOV; the angular area in the virtual world that the user can see at any instant in time, or the FOV of the virtual camera).

- Field of regard (FOR; the angular area surrounding the user within which the virtual world is displayed).

In the present study, we investigate which of these components had a positive effect on procedure memorization. We hypothesized that an SFOV that is matched to the physical world, in combination with a high FOV and a high FOR, would result in a high level of spatial understanding, thus facilitating memorization. Further, unlike the medical procedure used by Sowndararajan et al. (2008), which was concrete and easily mapped to a virtual world, our experiment was designed to use a more abstract procedure type. By using an abstract procedure, we were able to reduce the influence of external knowledge and experience on task familiarity and user strategy. Additionally, because VR applications for education and training would be of little use if the learned knowledge could only be used within the virtual training environments, we also tested the transfer of learning from a VE to the real world.

\subsection{Experimental Design}

We varied FOV, SFOV, and FOR as between-subjects independent variables in this experiment. FOV had two levels: low $\left(60^{\circ}\right)$, and high (nearly $\left.180^{\circ}\right)$. We used physical blinders on goggles to restrict the FOV in the low FOV conditions, and nonblinded glasses for the high FOV conditions (see Figure 1). SFOV had two levels: matched (virtual camera has the same FOV as the user, $90^{\circ}$ for each screen), and unmatched (virtual camera has an $\mathrm{FOV}$ of $135^{\circ}$ for a screen). FOR also had two levels: low $\left(90^{\circ}\right.$, using one projection screen), and high $\left(270^{\circ}\right.$, using three projection screens surrounding the user).

Overall, then, there were eight possible between-subjects conditions. However, we did not test conditions with an unmatched SFOV and a high FOR, as this would have resulted in severe distortions across the three screens. This left us with six between-subjects conditions (see Table 1).

In practical terms, the components that we varied enabled us to simulate the conditions of widely-used VR 


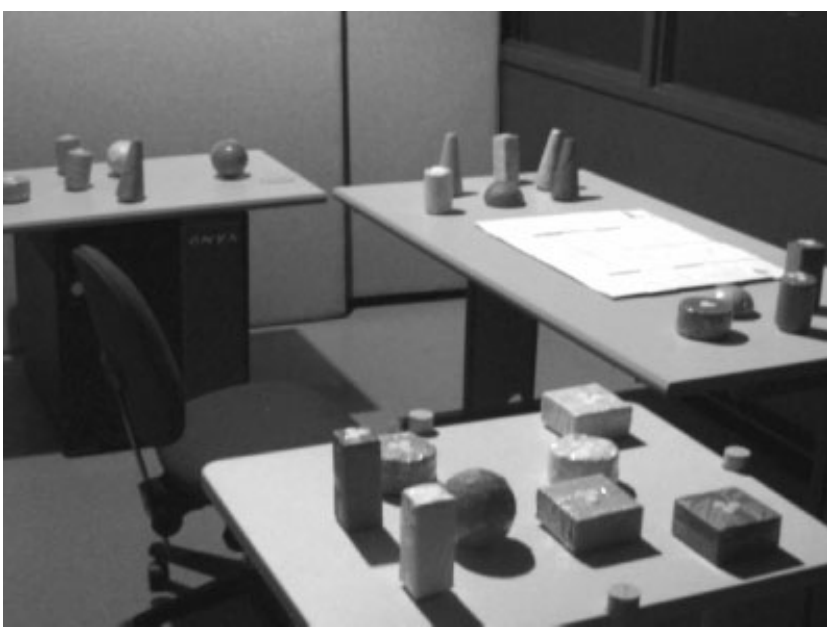

Figure 2. Real room used for initial memory test and real-world assessment conditions.

systems, such as the CAVE (high FOR, high FOV, matched SFOV), large-screen displays (low FOR, high FOV, matched SFOV), HMDs (high FOR, low FOV, matched FOV), and desktop displays (low FOR, low FOV, unmatched SFOV). Thus, our results can guide the choice of display system to use for educational applications that involve procedure memorization.

We also had two within-subjects independent variables: assessment environment (AE) and object consistency (OC). AE refers to the setting in which the assessment (or recall) phase was performed. Assessments were either completed in the physical world (Figure 2) or in the virtual world (Figure 3 ). In the virtual world conditions, the highest level of immersion (high FOV, matched SFOV, and high FOR) was always used for the assessment phase. AE was used to determine whether learning transferred to the real world. OC refers to the spatial locations of the objects in the environment during the assessment: objects could be in the same locations as they were during the learning phase (maintaining object consistency), or they could be in different locations (having no object consistency). OC was used to test whether participants relied on the exact spatial location of the objects for recall, or whether they could remember the procedure accurately even when the objects were moved. We hypothesized that neither AE nor OC would have a significant effect on the results because we

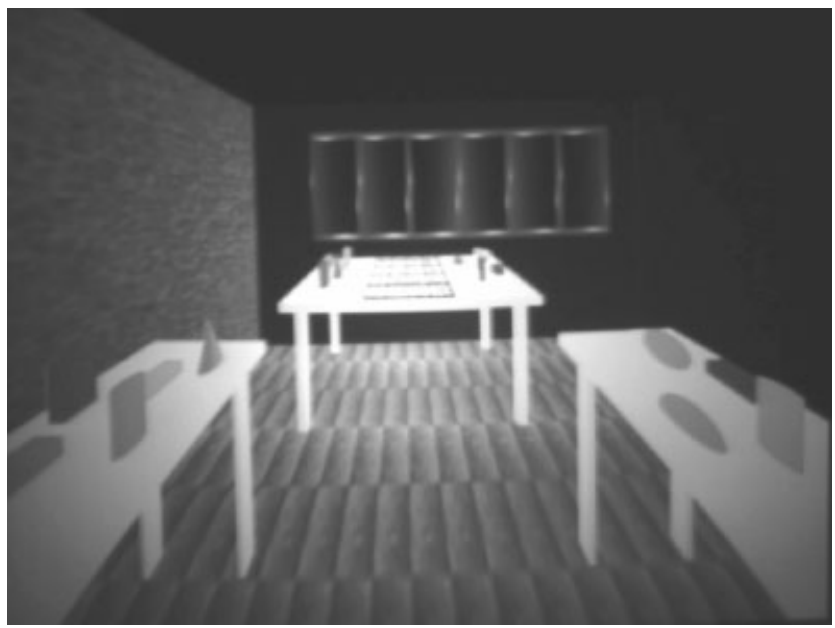

Figure 3. Virtual room, shown here with distorted (unmatched) SFOV.

believed that participants' recall would be based on their memories of the VE in which they learned a procedure, rather than on their surroundings during recall.

Thus, there were four within-subjects conditions. Each participant was placed in one of the six betweensubjects groups and performed one trial in each of the four within-subjects conditions. The four trials required participants to learn different procedures, but each procedure had an equivalent level of complexity; thus we do not consider procedure as an independent variable.

As in our earlier study (Sowndararajan et al., 2008), the dependent variables were the time to complete the assessment phase and the number of errors in the assessment phase. An error was counted every time the participant specified a step of the procedure incorrectly, up to a maximum of 10 errors per step. After 10 errors on a step, the experimenter provided that step of the procedure to the participant.

\subsection{Participants}

Forty-one voluntary, unpaid participants took part in the experiment. Twenty-five of the participants were male and the mean age was 22. Eight participants had used immersive VR previously, while 18 had video game experience. Each participant was screened with an initial memory test (see section 3.4) in the real world; five 
Table I. Levels of Immersion Tested in the Experiment*

$\begin{array}{llll}\text { Matched SFOV } & \text { Unmatched SFOV } & \text { Low FOV } & \text { High FOV } \\ \text { Low FOR } & & \end{array}$

\footnotetext{
* The figures are top-down views of the CAVE display. The triangles represent screens that were turned on in each condition to control FOR, with the size of each triangle indicating the SFOV of the virtual camera. The dotted arcs in the center represent the user's physical FOV.
}

participants scored below a predefined threshold on this test and did not complete the remainder of the experiment. The remaining 36 participants were assigned to the six between-subjects groups so that each group had six participants and the groups had approximately equal average scores on the initial memory test.

\subsection{Experimental Procedure}

Before beginning the experiment, participants gave their informed consent and answered a demographic questionnaire. They then performed the initial memory test, which involved the memorization of an eight-step procedure (similar to those used in the main experiment), with both learning and assessment taking place in a real-world setting.

As in the previous memorization experiment (Sowndararajan et al., 2008), the participant's task was to memorize a multistep procedure. Each step consisted of an object, a source location, and a destination. Each object was a 3D geometric solid, identifiable by its shape (box, tall box, sphere, hemisphere, cone, tall cylinder, or wide cylinder) and its color (red, green, blue, yellow, or pink). The shapes and colors were chosen so that each was distinct enough to be easily distinguished from the others. The participant was centered in an environment containing three tables-one on the left, one on the right, and one directly in front of the participant. To control the assessment environment variable, the procedure was performed in either a virtual room (within a CAVE) or within a real, physical room. The virtual world was modeled to look like a real room so that both versions of the environment shared the same setup.

At the start of each procedure, 28 objects were spread out among the tables (Figures 2 and 3 ). An object's source location was the table on which it was initially sitting. The front table also held a white $4 \times 4$ grid with numbered squares to serve as the target destination areas. In each step of the procedure, an object was moved from its initial location to a specific, numbered square of the grid (as shown in Figure 4). For example, a step might be: "Move the yellow sphere from the right table to position number 6." For some steps, the object was to be placed on top of an object that had previously been moved to the grid. An example of a description of such a step might be: "Move the red hemisphere from the left table and place it on top of the tall yellow cylinder at position number 11." By these types of instructions and the corresponding visualization, participants were tasked with remembering which object was moved 


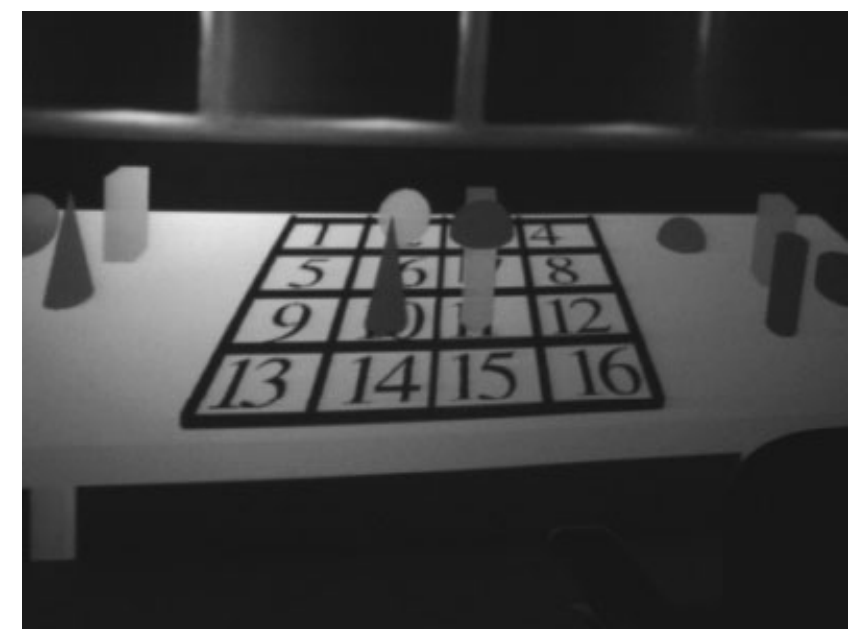

Figure 4. Target grid in the virtual environment.

and where it was moved to for each step of the procedure.

Each trial consisted of learning, practice, and assessment phases. In the learning phase, the experimenter first identified the objects that would be used in the procedure, and then explained each step of the procedure. The participant was taught to verbally describe the steps of the procedure in specific terms that included the object color, object shape, and destination location on the grid. While the experimenter described each step, that step was shown visually in the VE (the object would be moved automatically from its source location to its destination, so that no interaction was required beyond a verbal description).

The practice phase allowed participants to rehearse, with the experimenter's assistance, the procedure from the learning phase. In this phase, we asked the participant to verbally describe the procedure, following the protocol from the learning phase. As the participant described each step correctly, that step was shown visually in the VE. If the participant made a mistake or could not recall the next step, the experimenter helped him or her to remember the correct step in the procedure.

In the assessment phase, participants were asked to recall the entire procedure in the assigned assessment environment (real or virtual) without any assistance from the experimenter. When the participant provided the current step correctly, the experimenter showed the next step visually (automatically in the VE and manually in the real world), and the scenario moved on to the next step.

The learning and practice phases were always conducted in the virtual world, with the level of immersion determined by the participant's group. The assessment phase was conducted in either the virtual world or the real world, and with objects in the same or different locations as compared to the learning and practice phases, depending on the values of $\mathrm{AE}$ and $\mathrm{OC}$ for the trial in question.

Before the main trials of the experiment, the participants completed two practice trials, for which the procedures consisted of four steps; the procedures consisted of eight steps in the four main trials. In the main trials, participants always encountered the four procedures in the same order, while the order of the four within-subjects conditions was counterbalanced using a Latin square.

Before each trial, participants were informed of the values of $\mathrm{AE}$ and $\mathrm{OC}$ for that trial. Participants were asked to concentrate on the task of memorization and to refrain from asking questions during a trial. We allowed participants to rotate the virtual world around its vertical axis during the learning and practice phases, but no other virtual navigation was allowed. Rotation was not necessary in the assessment phase, since it was always performed either in the VE with the highest level of immersion, or in the real world, where all objects were visible without rotation.

\subsection{Apparatus}

We used a three-screen (front wall and two side walls) Fakespace CAVE to implement all six betweensubjects conditions. Each screen was $10 \mathrm{ft}$ wide and $9 \mathrm{ft}$ high. Screens were rear-projected, using $1280 \times 1024$ Electrohome CRT projectors. In the high FOR conditions, all three screens were used, while the low FOR condition used only the front screen. Participants held an Intersense IS-900 wand, using the analog joystick to rotate the virtual world around its vertical axis. We did not track the participants' head or hands (since participants were stationary and could still look to the left or 
Table 2. Results with Respect to Time,* Given as Least Square Means

\begin{tabular}{llllll}
\hline & \multicolumn{2}{l}{ Matched SFOV } & & \multicolumn{2}{l}{ Unmatched SFOV } \\
\cline { 2 - 3 } \cline { 5 - 6 } & Low FOV & High FOV & & Low FOV & High FOV \\
\hline Low FOR & 78.76 & $52.73^{* *}$ & & 84.66 & 81.91 \\
High FOR & $49.54^{* *}$ & $45.53^{* *}$ & & & \\
\hline
\end{tabular}

* Times are in seconds.

${ }^{* *}$ Conditions in these cells are significantly faster than other conditions.

Table 3. Results with Respect to Errors, Given as Least Square Means

\begin{tabular}{llllll}
\hline & \multicolumn{2}{l}{ Matched SFOV } & & \multicolumn{2}{l}{ Unmatched SFOV } \\
\cline { 2 - 3 } \cline { 5 - 6 } & Low FOV & High FOV & & Low FOV & High FOV \\
\hline Low FOR & 78.76 & $52.73^{*}$ & & 84.66 & 81.91 \\
High FOR & $49.54^{*}$ & $45.53^{*}$ & & \\
\hline
\end{tabular}

*Conditions in these cells are significantly more accurate than all other conditions.

right in the high FOR condition without head tracking, and since no direct interaction with the environment was needed), nor did we use stereoscopic graphics (the stereo glasses would have limited the range of physical FOV we could test). The environment was rendered using DIVERSE (Kelso, Satterfield, Arsenault, Ketchan, \& Kriz, 2003).

Participants were seated on a chair in the center of the CAVE and we varied the height of the chair so that each participant's head was at the same level. This allowed us to control the viewing perspective for all participants, regardless of different individual heights.

The virtual world was modeled to look like a real room that was physically adjacent to the CAVE (Figures 2 and 3 ). This ensured that participants could immediately transition between phases of each trial without significant lapses in time.

\subsection{Results}

We performed a mixed analysis of variance (ANOVA) on both time and errors. Note that $p$-values for the error analysis are only approximate, since the number of errors is not a continuous variable or neces- sarily normally distributed. Even after consulting with statisticians, we were not able to identify any nonparametric tests that could do the analysis due to our complex experimental design. In cases like these, ANOVA is considered the best approximation. As we will show, our results for the error metric are nearly identical to the results for the time metric, increasing our confidence that our analysis of the error metric is reasonable. More importantly, our effect sizes are large relative to variability, and are clearly meaningful in this context.

As we hypothesized, the within-subjects factors had no significant effect. AE was neither significant for time, $F(1,103)=0.037, p=.849$, nor errors, $F(1,103)=$ $0.862, p=.355$. Similarly, OC was neither significant for time, $F(1,103)=0.228, p=.634$, nor errors, $F(1,103)=0.364, p=.547$.

Table 2 shows the least squares means for time in all six between-subjects conditions, while Table 3 gives the same information for errors. We found main effects of SFOV for both time, $F(1,30)=15.85, p<.001$, and errors, $F(1,30)=123.81, p<.0001$. Matched SFOV resulted in less recall time $(M=59.99 \mathrm{~s})$ and fewer errors $(M=1.56)$ than unmatched SFOV $(71.05 \mathrm{~s}$ and 5.40 errors). 


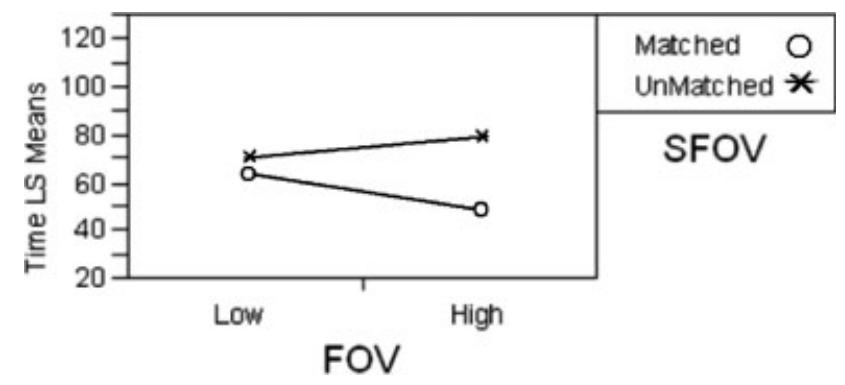

Figure 5. Interaction between SFOV and FOV over time. The combination of matched FOV with high FOV was significantly faster than the other three combinations.

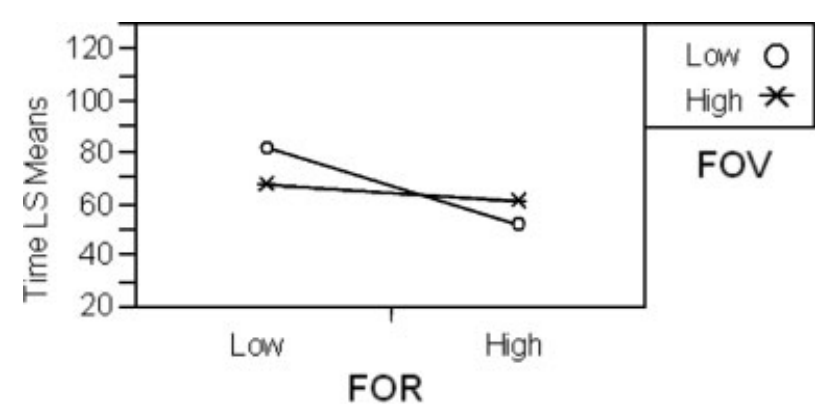

Figure 6. Interaction between FOV and FOR over time. The combination of low FOV and low FOR was significantly slower than the other three combinations.

Our analysis also found main effects of FOR for both time, $F(1,30)=17.09, p<.001$, and errors, $F(1,30)=$ $13.35, p<.001$. The high FOR conditions had lower assessment time (58.35 s) and fewer errors $(2.10)$ than the low FOR conditions ( $72.69 \mathrm{~s}$ and 4.86 errors).

We did not find a main effect of FOV on time, $F(1,30)=0.589, p=.449$, although high FOV conditions did have a faster average time (60.06 s) than low FOV (70.99 s). There was a main effect of FOV on errors, $F(1,30)=4.31, p<.05$, with high FOV resulting in fewer errors (2.72) than low FOV (4.21).

We found a significant interaction between SFOV and FOV for the time metric, $F(1,30)=6.982, p<.02$, shown in Figure 5. A post hoc analysis using a Tukey HSD test showed that the combination of matched SFOV with high FOV resulted in significantly faster recall than the other three combinations of these two variables.

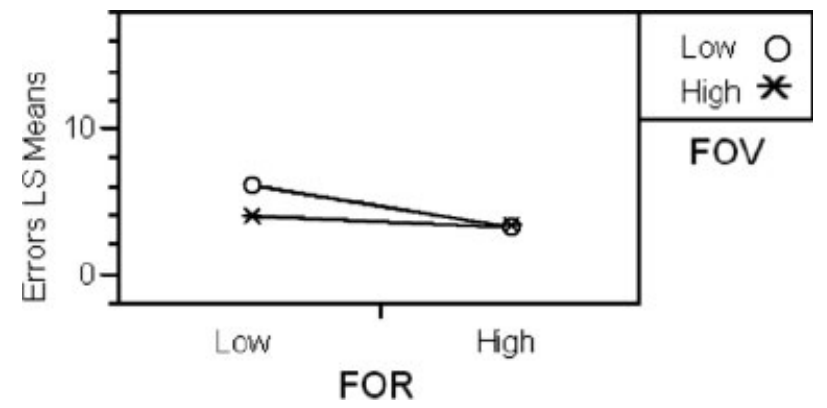

Figure 7. Interaction between FOV and FOR with respect to errors. The combination of low FOV and low FOR resulted in significantly more errors than the other three combinations.

There was also a significant interaction between FOV and FOR for both time, $F(1,30)=6.24, p<.02$, and errors, $F(1,30)=4.31, p<.05$, as shown in Figures 6 and 7 . Tukey HSD tests revealed that the combination of low FOV and low FOR was significantly slower and resulted in significantly more errors than the other three combinations of these variables.

We also ran post hoc Tukey tests to compare the six between-subject conditions with one another. Table 2 shows the results for time, while Table 3 shows the results for errors. In both cases, the same three conditions formed a separate group with better performance than the other conditions; these conditions all had a matched SFOV and either a high FOR or a high FOV, or both.

\section{Discussion}

All of the tested components of immersion contributed to performance differences. In this section, we interpret the results and discuss their implications for educational VR. In the research of learning in 3D environments, we consider the distinction between spatial and nonspatial types of information to be a nontrivial issue.

\section{I Interpreting the Results}

As we hypothesized, the assessment environment had no statistically significant effect on recall time or 
accuracy in our procedure memorization task. This means that the procedure, memorized in the virtual world, could be recalled in the real world just as quickly and with the same level of accuracy. Thus, the learning that was done in the virtual world was transferred to the real world. This has important implications for the use of immersive VR technologies for conceptual learning, since the learning would be useless unless it could be used outside the virtual world. Our experiment does not show, however, that learning in a virtual environment is as effective as learning in the real world; we leave this for future work. Regardless, the advantages of virtual environments (flexibility, control, the ability to display scenes not possible in the real world) would still make them attractive for learning applications.

Ideally, learners want to be able to recall and use the learned information in any environment-even those without any of the original cues of the practice environment. We found that object consistency (whether objects were in the same positions during assessment as they were in training and practice) had no effect on either recall time or accuracy. This means that participants did not rely (exclusively) on the presence of the same cognitive aid used during training/practice when recalling the memorized procedure. In other words, they appear to have been able to recall the procedure in its abstract form, rather than simply remembering the spatial locations or necessary movements of the objects. Recalling the procedure in exactly the same environment did not increase performance; or, said another way, recalling the procedure in a jumbled version of that environment did not decrease performance. While this result serves as evidence that participants were not relying on the specifics of the practice environment, even assessment environments with jumbled object arrangements still provided environmental stimuli very similar to that of the learning environment. A follow-up study is needed to verify that similar results would be obtained with recall taking place in an environment with no cognitive aids.

Higher levels of immersion during learning and rehearsal (i.e., matched SFOV, high FOV, and high FOR) all significantly improved recall accuracy, while both matched SFOV and high FOR significantly reduced recall time. These results also matched our hypotheses (with the one exception that high FOV did not reduce recall time significantly). As we described earlier, we believe that these increases in participants' ability to memorize procedures relate to the richness and quality of the spatial cues provided by the VE. The higher levels of immersion provided richer and better spatial cues, leading to increased spatial understanding, and allowing participants to use a spatial memory strategy (similar to the method of loci) for memorizing the procedure. Our experiment does not prove this assertion, but our results are consistent with this overall hypothesis.

Furthermore, we saw that various combinations of components of immersion produced better results than others. The significant interaction between SFOV and FOV (Figure 5) showed that both a matched SFOV and a high (unrestricted) FOV were necessary for achieving a significant decrease in recall time. The significant interactions between FOV and FOR (Figures 6 and 7) reveal that performance was significantly reduced when both FOV and FOR were at low levels.

These findings were reinforced by the post hoc comparison of all six levels of immersion (Tables 2 and 3 ). For both time and accuracy, the best conditions were those that had a matched SFOV and either a high FOV or a high FOR, or both. Matched SFOV seems, therefore, to be the most important component (among those we tested) for producing good recall performance, which partially explains the experiment results of Sowndararajan et al. (2008). In that earlier experiment, we were forced to use an unmatched SFOV on the laptop display to allow the user to see more of the virtual world. But matched SFOV by itself was not enough to produce the best performance in our experiment; high FOV and/or high FOR were also needed to provide sufficient spatial cues.

Although the highest level of immersion (matched SFOV, high FOV, high FOR) produced the lowest recall times and highest accuracy rates in absolute terms, the post hoc tests reveal that performance in this condition was not significantly different from two other conditions (the matched SFOV, high FOV, and low FOR condition with $p=.30$; and matched SFOV, low FOV, and high FOR condition with $p=.14$ ). With a greater number of 
subjects, the difference might have been significant, but this result still has important implications for real-world systems. In terms of widely-used VR displays, the condition with matched SFOV, high FOV, and high FOR corresponds to a CAVE-like system. The condition with matched SFOV, high FOV, and low FOR corresponds roughly to a large-screen display. And the condition with matched SFOV, low FOV, and high FOR has characteristics similar to most HMDs. From a practical point of view, our experiment seems to suggest that while highend VR systems (CAVE-like displays) may provide the best overall performance, lower-cost VR systems with moderate levels of immersion (large-screen displays and HMDs) can still result in significant performance gains over less immersive displays for the task of procedure memorization. This is important for determining which type of system is necessary for educational and training purposes. If a more affordable, lower-end system can afford the same (or nearly the same) benefits as considerably more expensive equipment, users could save a great deal on system costs. If more affordable immersive technology offers clear educational advantages, it can become a much more practical option for common use.

\subsection{Spatial Advantages of Higher Immersion for Procedure Memorization}

Though our work provides evidence that higher levels of immersion can help improve performance on certain memorization tasks, it is still unknown if these benefits apply to a large variety of learning and training tasks. We have hypothesized that the benefits are derived from the enhanced spatial cues offered by more immersive VR displays. In this study, the matched SFOV conditions permit a more natural perception of the $3 \mathrm{D}$ space than the distorted, unmatched SFOV, making it easier to perceive and understand the object arrangements and positional changes. A matched SFOV supports a more natural interpretation of the static depth cues that we are accustomed to using automatically in our daily lives (with occlusion, relative size, height relative to the horizon, and linear perspective being the significant cues available for this experimental task). The high FOV con- ditions allowed participants to utilize peripheral vision to view more of the objects at once without view rotation, making it possible to observe both an object's initial position on one of the tables as well as its final position on the target grid without rotating. For each step of the sequence, the overall change in the scene could be observed in both object positions without requiring significant view adjustments. We believe this aided the ability to remember the construction procedure of the final object arrangement on the grid. Finally, the high FOR allowed participants to use physical head rotations rather than requiring virtual rotation to view the entire scene. Past work has demonstrated benefits to physical, rather than virtual, navigation for certain tasks (Ball, North, \& Bowman, 2007; Chance, Gaunet, Beall, \& Loomis, 1998; Pausch, Proffitt, \& Williams, 1997). In our experimental task, the physical rotation allowed by the higher FOR not only enabled the use of proprioceptive cues to help maintain an egocentric model of the environment during the learning and practice phases, but may have also provided the added benefit of motor memory cues to aid memorization and recollection (Cohen, 1989).

We note that it is true that the more immersive conditions in our experiment more closely matched the assessment environments (either the real, physical room or the VE with high FOV, matched SFOV, and high FOR). As such, one possible explanation for superior performance with the more immersive learning and practice conditions could have been that participants performed better because the learning environment was more similar to the assessment environment. However, in our previous study (Sowndararajan et al., 2008), the recall assessment was always done in the same conditions as the learning and practice phases-and the study still revealed better performance when the combination of matched SFOV, higher FOR, and higher FOV was provided for the procedure memorization task. Because the medical procedure memorization task of the previous study was so similar to the abstract procedure memorization of the currently presented study, we believe that rather than stemming from similarities in the learning and assessment environments, the performance benefits are due to the spatial advantages provided by the FOV, FOR, and SFOV themselves. 


\subsection{Distinguishing Between Spatial and Nonspatial Information}

If it is true that the performance improvements are due to better spatial cues, then does it also follow that the benefits of higher immersion only apply to tasks or procedures with components that are spatial in nature? Both the medical procedure memorization task and the task of the current study certainly fall into this category, requiring participants to remember objects arranged in space and their new locations during the procedures. On the other hand, our tasks also required participants to learn a great deal of nonspatial information. For example, in the medical procedure task, participants learned the order in which tools and supplies needed to be applied to successfully conduct the procedure. In the experiment reported in this paper, participants did not just memorize initial and final locations of objects, but also had to remember which objects were used (out of a greater number of objects that were not involved in the procedure) and in what order they were to be moved. Granted, it would be difficult to dissociate this information entirely from the spatial components of the tasks, but nevertheless, the information itself was not inherently spatial.

Though our hypothesis that the performance improvements were due to greater spatial cues is partially supported by past studies showing that additional spatial cues can aid memorization (Hess et al., 1999), we do not clearly understand how participants took advantage of the spatial cues in our study. We suspect that, even without special instruction, participants were able to use these cues to improve the memorization strategies they used for the task. One strategy that may have been used involves memorizing the final object arrangement on the grid and then trying to remember the order in which the individual objects were added to form the arrangement. In this potential strategy, the object arrangement is remembered as a single $3 \mathrm{D}$ structure that is used as a memory aid for the individual steps of the procedure. Another likely strategy involves remembering the individual steps of the procedure, focusing on how the layout of surrounding objects changed as the procedure progressed. Of course, these strategies could also have been used in conjunction, taking advantage of the spatial layout of the learning environment in addition to the structure of final object formation. Unfortunately, we were unable to accurately categorize participant strategies used for the memorization task, as the procedure's complexity and multitude of information types made it difficult for participants to effectively identify and describe their own strategies. A follow-up investigation is needed to determine whether spatial strategies were commonly used to support memorization or if there is another explanation for why increased levels of immersion led to better performance.

\subsection{Designing to Support the Learning of Both Spatial and Nonspatial Information}

Many of the procedures and activities that commonly take advantage of VR applications (e.g., medical training, automotive assembly, disaster rescue training) are heavily based upon inherently spatial information, and many successful educational VR applications teach concepts that are based on physical phenomena or spatial representations. For instance, the MaxwellWorld application (Dede, Salzman, Loftin, \& Sprague, 1999), which was shown to provide significantly stronger conceptual understandings of electrostatic fields compared to more traditional instructional methods, allowed students to learn these concepts through interactive explorations within virtual space. As another example, the Construct3D application showed promise for assisting the learning of 3D geometric structures (Kaufmann, Schmalstieg, \& Wagner, 2000). Perhaps these applications were successful because they were so strongly based on spatial information, allowing students to benefit from the additional spatial cues offered by immersive technology.

On the other hand, many educational topics (e.g., foreign languages, mathematics, history) may not have clear physical or spatial representations from which their core concepts can be learned. We are challenged with investigating whether the learning of such nonspatial concepts can still benefit from immersive technology. As at least some of the information learned in our experimental tasks was nonspatial in nature, we believe that it can. We 
are left with a question of design; that is, how can abstract information be spatially presented to take advantage of the benefits of immersive technology?

This question exposes numerous potential design factors for further consideration when developing educational applications. It is not well understood how spatial cues can be used to support efficient learning. Is simply presenting information in spatial layouts, displaying information in different locations, enough to improve performance on certain learning tasks? Researchers have hypothesized that spatial information may be stored in a different area of working memory than some other types of information (Baddeley, 1998). Past studies have provided evidence that it is possible to take advantage of these different memory stores to improve task efficiency by relying on more than one information type (e.g., Duff \& Logie, 2001; Wickens \& Liu, 1988). Similarly, by presenting information in spatial layouts, learners may be able to take advantage of spatial offloading or use locations as redundant cues in order to improve learning efficiency. As we described earlier, the method of loci (Yates, 1974) uses just this type of spatial indexing to allow the memorization and recall of large amounts of nonspatial information.

But is arranging information in space enough, or is intentional design of the spatial organization required to achieve the benefits of such a presentation? This is another issue to be investigated. Perhaps any arbitrary spatial arrangement will not actually be helpful unless the learner can perceive some meaningful organization of the information. Further, if this is the case, will learners be dependent on the provided organizational design for meaning, or can they construct their own meaning of the information space through environmental interactivity? Numerous past researchers have pointed to interactivity (e.g., Wickens, 1992; Salzman et al., 1999) as a highly valuable feature of educational VR. Rather than using interaction purely for constructivist exploration, interactivity can be used to allow learners to control how they experience information spatially. For example, navigation, a basic and common type of interaction, can be used to control the order in which different pieces of information are encountered, as well as the duration and frequency of viewing. We suspect that such increased level of control may be particularly beneficial for learning abstract types of information within a VE. Bowman et al. (1999) found evidence of learning gains with a virtual zoo application for habitat design education that allowed users to navigate freely to view textual information coupled with various habitat components spread throughout the environment. While some of the presented information was clearly spatial in nature due to its relationship to the design of physical spaces, other supplemental information was factual and nonspatial. We are interested in investigating whether interaction-even that as simple as navigation-could impact the effectiveness of learning through spatial designs.

\subsection{Beyond Memorization}

Although a relatively simple form of learning, memorization is teaching us a great deal about how display features can affect performance. In addition to the study of immediate recall, longer-term retention issues are also important for consideration. What will learners remember weeks or months after learning nonspatial information presented along with primarily spatial cues? It is possible that the extra spatial cues could aid the development of a stronger mental organization of the nonspatial material, leading to greater retrieval efficiency. Conversely, as an undesirable outcome, the spatial information might interfere with the retrieval of other information of interest.

Even looking beyond memorization and simple recall, it is still unknown how other types of higher-level cognitive processing activities might benefit from increased levels of immersion. Many previously developed educational VR applications were designed to help students not only to remember presented information, but also to understand complex principles that are considered difficult to comprehend. For example, the three virtual worlds of ScienceSpace (Dede et al., 1996) were designed to allow students to explore molecular structures, investigate basic principles of Newtonian physics, and experiment with electrostatic fields. Another application, the NICE garden (Roussos et al., 1999), was designed to help students understand plant life cycles and their relationships to agents of nature, while 
Quarles, Lampotang, Fischler, Fishwick, and Lok (2008) employed a mixed reality system to help anesthesiology students connect their abstract mental models of equipment functionality to the actual workings of the physical, real-world machines. Attempting to support problem-solving activities as well, the exercises of the Virtual Playground (Roussou et al., 2006) were not only meant to help students to better understand numerical fractions, but also how to think about them when solving mathematical fraction problems.

Achieving a more complete body of knowledge of the effects of different immersion components on learning will require evaluation of learning activities more complicated than simply memorization and recollection. Future studies should consider investigating the understanding and application of learned knowledge. Evaluating such higher orders of thinking for a variety of controlled experimental tasks can provide a great deal of design knowledge about the types of learning activities that can best be supported by immersive VR systems. Unfortunately, the design of such controlled experiments on higher-level cognition is not trivial. Highly controlled studies based on more generic tasks help produce generalizable results and guidelines for developing specific, more refined applications, but evaluation of the effectiveness of these applications is just as important for testing the developed guidelines and identifying additional issues that may be missed in controlled studies.

\section{Conclusion}

Applications of immersive VR to conceptual learning and training applications have been proposed, but there has been little evidence to support the assertion that immersive VR systems can produce better learning. Our experiment on the effects of level of immersion on procedure memorization does not fully answer the question, but it does provide empirical evidence that higher levels of immersion can produce a measurable improvement in the performance of an abstract mental activity.

In addition, we have shown that a finer-grained view of immersion as a multidimensional continuum can result in a deeper understanding of its effects. In our experiment, because we studied three independent components of immersion, we were able to say not only that higher levels of immersion resulted in better performance, but also that these benefits are due to increased FOV, increased FOR, and matched SFOV. Further, conditions corresponding to lower-cost VR systems offered statistically significant performance improvements over conditions with lower levels of immersion. Significant benefits for procedure memorization can be obtained even without using the highest possible level of immersion.

Clearly, much future work is needed. We noted in the previous section the need for follow-up studies to compare learning in a VE with learning in the real world, and to investigate how well learners recall a procedure in the absence of any (physical or virtual) cognitive aid. Additional experimentation is also required to determine whether participants are indeed using a spatial memorization strategy, as we surmise, and whether they use this strategy more often when they learn in higher levels of immersion. We are also interested in exploring the importance of utilizing a spatial layout for information presentation, as we hypothesize that the effectiveness of memorization techniques may be enhanced through additional spatial cues. We could also investigate whether participants with higher spatial ability, or those who gain more spatial understanding of the VE, perform better in the procedure memorization task.

Beyond procedure memorization, empirical evidence of the effects of immersion (and its components) is needed for other abstract mental activities, and for higher-level conceptual learning processes. Finding appropriate measures and procedures for such experiments, however, will be a difficult challenge.

\section{References}

Allison, D., \& Hodges, L. F. (2000). Virtual reality for education? Proceedings of the ACM Symposium on Virtual Reality Software and Technology, 160-165.

Arns, L., Cook, D., \& Cruz-Neira, C. (1999). The benefits of statistical visualization in an immersive environment. Proceedings of the IEEE Virtual Reality Conference, 88.

Baddeley, A. (1998). Working memory. Comptes Rendus de l'Académie des Sciences, Ser. III, Sciences de la Vie, 321(2-3), 167-173. doi:10.1109/VR.1999.756938 
Ball, R., North, C., \& Bowman, D. A. (2007). More to improve. Proceedings of the SIGCHI conference on human factors in computing systems-CHI'07, p. 191. San Jose, California.

Bloom, B. S., Englehart, M. D., Furst, E. J., Hill, W. H., \& Krathwohl, D. R. (1956). Taxonomy of educational objectives: The classification of educational goals. Handbook I: Cognitive domain. New York: David McKay.

Botden, S., Buzink, S., Schijven, M., \& Jakimowicz, J. (2007). Augmented versus virtual reality laparoscopic simulation: What is the difference? World Journal of Surgery, 31(4), 764-772. doi: 10.1007/s00268-006-0724-y

Bowman, D. A., Hodges, L. F., Allison, D., \& Wineman, J. (1999). The educational value of an information-rich virtual environment. Presence: Teleoperators and Virtual Environments, 8(3), 317-331. doi:10.1162/105474699566251

Bowman, D. A., \& McMahan, R. (2007). Virtual reality: How much immersion is enough? Computer, 40(7), 36-43. doi:10.1109/MC.2007.257

Bowman, D. A., Sowndararajan, A., Ragan, E. D., \& Kopper, R. (2009). Higher levels of immersion improve procedure memorization performance. Proceedings of Joint Virtual Reality Conference 2009. doi:10.2312/EGVE/JVRC09/ 121-128

Brooks, B. M., Attree E. A., Rose F. D., Clifford B. R., \& Leadbetter, A. G. (1999). The specificity of memory enhancement during interaction with a virtual environment. Memory, 7, 65-78. doi:10.1080/741943713

Brooks, F. (1999). What's real about virtual reality? Computer Graphics and Applications, IEEE, 19(6), 16-27. doi:10.1109/38.799723

Chance, S. S., Gaunet, F., Beall, A. C., \& Loomis, J. M. (1998). Locomotion mode affects the updating of objects encountered during travel: The contribution of vestibular and proprioceptive inputs to path integration. Presence: Teleoperators and Virtual Environments, 7(2), 168-178. doi:10.1162/105474698565659

Cohen, R. (1989). Memory for action events: The power of enactment. Educational Psychology Review, 1(1), 57-80. doi:10.1007/BF01326550

Dede, C., Salzman, M. C., \& Loftin, R. B. (1996). ScienceSpace: Virtual realities for learning complex and abstract scientific concepts. Proceedings of the 1996 Virtual Reality Annual International Symposium (VRAIS'96), 246. doi:10.1109/VRAIS.1996.490534

Dede, C., Salzman, M. C., Loftin, R. B., \& Sprague, D. (1999). Multisensory immersion as a modeling environment for learning complex scientific concepts. Modeling and simulation in science and mathematics education (pp. 282-319). Berlin: Springer Verlag.

Duff, S. C., \& Logie, R. H. (2001). Processing and storage in working memory span. The Quarterly Journal of Experimental Psychology Section A: Human Experimental Psychology, 54(1), 31. doi:10.1080/02724980042000011.

Ericsson, K. A. (2003). Exceptional memorizers: Made, not born. Trends in Cognitive Sciences, 7(6), 233-235. doi:10.1016/S1364-6613(03)00103-7

Hess, S. M., Detweiler, M. C., \& Ellis, R. D. (1999). The utility of display space in keeping track of rapidly changing information. Human Factors: The Journal of the Human Factors and Ergonomics Society, 41(2), 257-281. doi:10.1518/ 001872099779591187

Johnsen, K., Dickerson, R., Raij, A., Lok, B., Jackson, J., Shin, M., et al. (2005). Experiences in using immersive virtual characters to educate medical communication skills. Proceedings of Virtual Reality. VR 2005, pp. 179-186. doi:10.1109/VR.2005.1492772

Johnson, A., Moher, T., Ohlsson, S., \& Leigh, J. (2001). Exploring multiple representations in elementary school science education. Proceedings of the Virtual Reality 2001 Conference, VR'01, 201. doi:10.1109/

VR.2001.913787

Johnson, W. L., \& Rickel, J. (1997). Steve: An animated pedagogical agent for procedural training in virtual environments. SIGART Bulletin, 8(1-4), 16-21. doi:10.1145/ 272874.272877

Kaufmann, H., Schmalstieg, D., \& Wagner, M. (2000). Construct3D: A virtual reality application for mathematics and geometry education. Education and Information Technologies, 5(4), 263-276. doi:1023/A:1012049406877

Kelso, J., Satterfield, S. G., Arsenault, L. E., Ketchan, P. M., \& Kriz, R. D. (2003). DIVERSE: A framework for building extensible and reconfigurable device-independent virtual environments and distributed asynchronous simulations. Presence: Teleoperators and Virtual Environments, 12(1), 19-36. doi:10.1162/105474603763835314

Kennedy, M. M. (1999). Approximations to indicators of student outcomes. Educational Evaluation and Policy Analysis, 21(4), 345-363. doi:10.3102/01623737021004345

Krathwohl, D. R. (2002). A revision of Bloom's Taxonomy: An overview. Theory Into Practice, 41(4), 212. doi:10.1207/ s15430421tip4104_2

Lidal, E. M., Langeland, T., Giertsen, C., Grimsgaard, J., \& Helland, R. (2007). A decade of increased oil recovery in vir- 
tual reality. IEEE Computer Graphics and Applications, 27(6), 94-97. doi:10.1109/MCG.2007.141

Mania, K., Robinson, A., \& Brandt, K. R. (2005). The effect of memory schemas on object recognition in virtual environments. Presence: Teleoperators and Virtual Environments, 14(5), 606-615. doi:10.1162/105474605774918769

Mania, K., Troscianko, T., Hawkes, R., \& Chalmers, A. (2003). Fidelity metrics for virtual environment simulations based on spatial memory awareness states. Presence: Teleoperators and Virtual Environments, 12(3), 296-310. doi:10.1162/105474603765879549

Pausch, R., Proffitt, D., \& Williams, G. (1997). Quantifying immersion in virtual reality. Proceedings of the 24th Annual Conference on Computer Graphics and Interactive Techniques, 13-18. doi:10.1145/258734.258744

Ponder, M., Herbelin, B., Molet, T., Schertenlieb, S., Ulicny, B., Papagiannakis, G., et al. (2003). Immersive VR decision training: Telling interactive stories featuring advanced virtual human simulation technologies. Proceedings of the Workshop on Virtual Environments 2003, 97-106. doi:10.1145/ 769953.769965

Quarles, J., Lampotang, S., Fischler, I., Fishwick, P., \& Lok, B. (2008). A mixed reality approach for merging abstract and concrete knowledge. Proceedings of the Virtual Reality Conference, VR '08, 27-34. doi:10.1109/VR.2008. 4480746

Roussos, M., Johnson, A., Moher, T., Leigh, J., Vasilakis, C., \& Barnes, C. (1999). Learning and building together in an immersive virtual world. Presence: Teleoperators and Virtual Environments, 8(3), 247-263. doi:10.1162/ 105474699566215

Roussou, M., Oliver, M., \& Slater, M. (2006). The virtual playground: An educational virtual reality environment for evaluating interactivity and conceptual learning. Virtual Reality, 10(3), 227-240. doi:10.1007/s10055-006-0035-5 Salzman, M. C., Dede, C., Loftin, R. B., \& Chen, J. (1999). A model for understanding how virtual reality aids complex conceptual learning. Presence: Teleoperators and Virtual Environments, 8(3), 293-316. doi:10.1162/ 105474699566242

Schuchardt, P., \& Bowman, D. A. (2007). The benefits of immersion for spatial understanding of complex underground cave systems. Proceedings of the 2007 ACM Symposium on Virtual Reality Software and Technology, 121-124. doi:10.1145/1315184.1315205

Slater, M. (2003). A note on presence terminology. Presence Connect, 3(3).

Sowndararajan, A., Wang, R., \& Bowman, D. A. (2008). Quantifying the benefits of immersion for procedural training. Proceedings of the 2008 Workshop on Immersive Projection Technologies/Emerging Display Technologies, 1-4. doi:10.1145/1394669.1394672

van Dam, A., Forsberg, A. S., Laidlaw, D. H., LaViola, J. J., \& Simpson, R. M. (2000). Immersive VR for scientific visualization: A progress report. IEEE Computer Graphics and Applications, 20(6), 26-52. doi:10.1109/38.888006

Ware, C., Arthur, K., \& Booth, K. S. (1993). Fish tank virtual reality. Proceedings of the INTERACT'93 and CHI'93 Conference on Human Factors in Computing Systems, 37-42. doi:10.1145/169059.169066

Ware, C., \& Mitchell, P. (2005). Reevaluating stereo and motion cues for visualizing graphs in three dimensions. Proceedings of the 2nd Symposium on Applied Perception in Graphics and Visualization, 51-58. doi:10.1145/ 1080402.1080411

Wickens, C. (1992). Virtual reality and education. Proceedings of the IEEE International Conference on Systems, Man and Cybernetics, 1992, Vol. 1, 842-847. doi:10.1109/ ICSMC.1992.271688.

Wickens, C. D., \& Liu, Y. (1988). Codes and modalities in multiple resources: A success and a qualification. Human Factors, 30(5), 599-616.

Yates, F. A. (1974). The art of memory. Chicago: University of Chicago Press. 